Journal of Advanced Research in Applied Sciences and Engineering Technology

\title{
MHD Flow and Heat Transfer of Double Stratified Micropolar Fluid over a Vertical Permeable Shrinking/Stretching Sheet with Chemical Reaction and Heat Source
}

\author{
Ansab Azam Khan ${ }^{1}$, Khairy Zaimi ${ }^{2},{ }^{*}$, Suliadi Firdaus Sufahani ${ }^{3}$, Mohammad Ferdows ${ }^{4}$ \\ School of Quantitative Sciences, UUM College of Arts \& Sciences, Universiti Utara Malaysia, UUM Sintok, Kedah Darul Aman, Malaysia. \\ Institute of Engineering Mathematics, Universiti Malaysia Perlis, Pauh Putra Campus, 02600 Arau, Perlis, Malaysia \\ Department of Mathematics and Statistics, Faculty of Applied Sciences and Technology, Universiti Tun Hussein Onn Malaysia, Johor, Malaysia \\ 4 Research Group of Fluid Flow Modeling and Simulation, Department of Applied Mathematics, University of Dhaka, Dhaka-1000, Bangladesh
}

Article history:

Received 7 May 2020

Received in revised form 13 July 2020

Accepted 22 August 2020

Available online 11 November 2020

\begin{abstract}
The present study analyses the magnetohydrodynamic (MHD) flow of a double stratified micropolar fluid across a vertical stretching/shrinking sheet in the presence of suction, chemical reaction, and heat source effects. The governing equations in the form of partial differential equations are transitioned into coupled nonlinear ordinary differential equations by means of similarity transformation. The numerical solutions are obtained with the aid of the boundary value problem bvp4c solver in the MATLAB software. Numerical results have been confirmed with the previous results for a certain case and the comparison is found to be in an excellent agreement. Results for related profiles and heat transfer characteristics are displayed through plots and tabulated for the governing parameters involved. It is found that the reduced skin friction coefficient and the local Nusselt number increase with the increasing chemical reaction and heat source parameters. The rising values of the chemical reaction parameter have increased the magnitude of the local Sherwood number. In contrary, the heat source parameter has the tendency to decrease the magnitude of the local Sherwood number.
\end{abstract}

Keywords:

double stratified; micropolar fluid; stretching/shrinking sheet; chemical reaction; heat source

Copyright @ 2020 PENERBIT AKADEMIA BARU - All rights reserved

\section{Introduction}

The theory and model of micropolar fluids were first introduced by Eringen [1-2] which describe the fluids with microstructure. This theory is able to explain the microscopic effects due to the local structure and micromotion of the fluid particles which are the main limitation in the classical NavierStokes equations. This model has been developed by considering an extra micro rotational momentum equation to the existing momentum equation. Lubricants, suspension solutions and

\footnotetext{
* Corresponding author.

E-mail address: khairy@unimap.edu.my

https://doi.org/10.37934/araset.21.1.114
} 
animal blood are examples of liquids that use the micropolar fluid model (Ahmad et al., [3]). In the book by Lukaszewicz [4], he explains the theory of the micropolar fluid and its two applications in the theories of lubrication and porous media. Over the past few decades, the study of micropolar fluids has gained a lot of attention because of its usage in many processes that are taking place in the industry.

In recent years, several boundary layer flow problems consider the double stratification effect in a micropolar fluid. Thermal and concentration stratifications occur due to temperature variations, concentration differences, or the presence of different fluids. The investigations on double stratification have attracted many researchers around the world due to the wide variety of applications in engineering such as thermal energy storage systems in solar ponds and the condensers of power plant involving the heat transfer from thermal sources (Srinivasacharya \& Upendar [5]). One of the related scholarly works on this topic is done by Srinivasacharya and Upendar [5]. In detail, Srinivasacharya and Upendar [5] have analyzed the effect of double stratification on free convection MHD micropolar fluid over a vertical permeable stretching/shrinking plate. The analysis showed that the velocity, skin friction coefficient, the local Nusselt number and the local Sherwood number decrease with the increasing magnetic parameter. In contrast, the temperature, concentration, and the local wall couple stress increase as the magnetic parameter increases. Besides that, another main finding indicates that the skin friction coefficient, Nusselt number and Sherwood number decreases and the wall couple stress increases as the thermal stratification parameter increases. It is also reported that the skin friction coefficient, Nusselt number and Sherwood number decreases and the wall couple stress increases as the solutal stratification parameter increases.

Later, Rashad et al., [6] focused on the thermal and physical properties of the features of mixed convection flow of micropolar fluid over a continuously moving isothermal vertical surface immersed in a double stratification (thermal and concentration) medium taking into consideration the chemical reaction and magnetic field effects. It is perceived that the skin friction coefficient, Nusselt and Sherwood numbers are enhanced with the increasing of material parameter. It is additionally observed that the local Nusselt number decreases with the chemical reaction parameter, whereas the skin friction coefficient and the local Sherwood number increase with it. Furthermore, it was also reported that the increase in the values of the thermal stratification parameter implies that the Sherwood number decreases while the opposite way occurred for the skin friction coefficient and the local Nusselt number. By contrast, it is identified that the skin friction coefficient rises as the solutal stratification parameter increased while both the local Nusselt and the local Sherwood numbers decrease with it.

Most recently, Khashi'ie et al., [7] investigated suction, magnetic field, and double stratifications effects on the mixed convection boundary layer flow over a stretching/shrinking sheet in micropolar fluid. They reported that dual solution exists in a certain range of suction and stretching/shrinking parameters. It is found that the velocity gradient, temperature gradient and concentration gradient increase with the increase in the suction parameter. Opposite trend is found with the addition of the material parameter where the velocity gradient, temperature gradient and concentration gradient decrease with it. Apart from that, they stated that the temperature of the fluid is enhanced with the thermal stratification parameter while the concentration is reduced with the increasing solutal stratification parameter. Also, it is further pointed out that both velocity and angular velocity have decreased with the increase in the magnetic parameter strength. Since then, there are numerous studies working on micropolar fluid under different physical conditions.

The impacts of heat source on the fluid flow problem over the stretching/shrinking sheet condition have been discussed in many studies. The heat source effects have significant applications in physics and engineering applications. The heat source effects evident in different flow problems 
are very useful in the particle deposition rate in nuclear reactors, electronic chips and semiconductor wafers (Ibrahim \& Suneetha [8]). Ibrahim and Suneetha [8] also mentioned the importance of study in the chemical reaction effect for example in an evaporation at the surface of a water body, energy transfer in a wet cooling tower and the flow in a desert cooler.

Motivated by the above mentioned study by Khashi'ie et al., [7], the present work seeks to analyze the heat and mass transfer of MHD micropolar fluid flow involving double stratification through a stretching/shrinking vertical sheet under chemical reaction and heat source effect. To the best of author's knowledge, no such studies are like the proposed study in the presence of heat source together with thermal and concentration stratifications. The governing equations in partial differential form are converted into a set of combined ordinary differential equations which are in nonlinear form. These resulting nonlinear ordinary differential equations are solved using bvp4c solver in MATLAB. The impacts of the governing parameters on the velocity, angular velocity, temperature, and concentration profiles as well as the skin friction coefficient, the local Nusselt number and the local Sherwood number have been plotted and analyzed wisely.

\section{Problem Formulation}

Consider the steady boundary layer flow and heat transfer of micropolar fluid over a linear stretching/shrinking vertical sheet in the presence of the magnetic field, suction, chemical reaction, and double stratification effects as illustrated in Figure 1. The stretching/shrinking sheet is assumed to have linear velocity $u_{w}(x)=a x$, a variable surface temperature $T_{w}(x)=T_{0}+A x$, concentration $C_{w}(x)=C_{0}+E x$ where $a, A$, and $E$ are constants with $T_{w}(x)>T_{0}$ and $C_{w}(x)>C_{0}$. The ambient temperature and concentration are assumed to be linearly stratified given by $T_{\infty}(x)=T_{0}+H x$ and $C_{\infty}(x)=C_{0}+P x$ where $H$ and $P$ are constants whilst $T_{0}$ and $C_{0}$ are initial ambient temperature and concentration. Under the boundary layer approximations, the corresponding boundary layers equation with the above-mentioned effects is (see Srinivasacharya \& Upendar [5], Rashad et al., [6], Khashi'ie et al., [7]):

$$
\begin{aligned}
& \frac{\partial u}{\partial x}+\frac{\partial v}{\partial y}=0 \\
& \rho\left(u \frac{\partial u}{\partial x}+v \frac{\partial u}{\partial y}\right)=(\mu+\kappa) \frac{\partial^{2} u}{\partial y^{2}}+\kappa \frac{\partial \omega}{\partial y}-\sigma_{M} B_{0}^{2} u+\rho g\left[\beta_{T}\left(T-T_{\infty}\right)+\beta_{C}\left(C-C_{\infty}\right)\right] \\
& \rho j\left(u \frac{\partial \omega}{\partial x}+v \frac{\partial \omega}{\partial y}\right)=\gamma \frac{\partial^{2} \omega}{\partial y^{2}}-\kappa\left(2 \omega+\frac{\partial u}{\partial y}\right), \\
& u \frac{\partial T}{\partial x}+v \frac{\partial T}{\partial y}=\alpha \frac{\partial^{2} T}{\partial y^{2}}+\frac{Q_{0}}{\rho C_{p}}\left(T-T_{\infty}\right) \\
& u \frac{\partial C}{\partial x}+v \frac{\partial C}{\partial y}=D \frac{\partial^{2} C}{\partial y^{2}}-k_{0}\left(C-C_{\infty}\right) .
\end{aligned}
$$


Here, $u$ and $v$ are the velocity components along $x$ and $y$ directions, $T$ and $C$ are the temperature and concentration of the fluid respectively, $\omega$ is the component of micro-rotation where the direction of rotation lies in the $x y$ - plane, $\rho$ is the fluid density, $\mu$ is the dynamic coefficient of the viscosity of the fluid, $\kappa$ is the vortex viscosity, $g$ is the gravitational acceleration, $\beta_{T}$ is the coefficient of thermal expansion, $\beta_{C}$ is the coefficient of solutal expansion, $B_{0}$ is the coefficient of the magnetic field, $j$ is the micro-inertia density, $\gamma$ is the spin-gradient viscosity, $\sigma_{M}$ is the magnetic permeability of the fluid, $\alpha$ is the thermal diffusivity, $Q_{0}$ is the uniform volumetric heat source and absorption, $C_{p}$ is the specific heat at constant pressure, $D$ is the molecular diffusivity and $k_{0}$ is the chemical reaction.

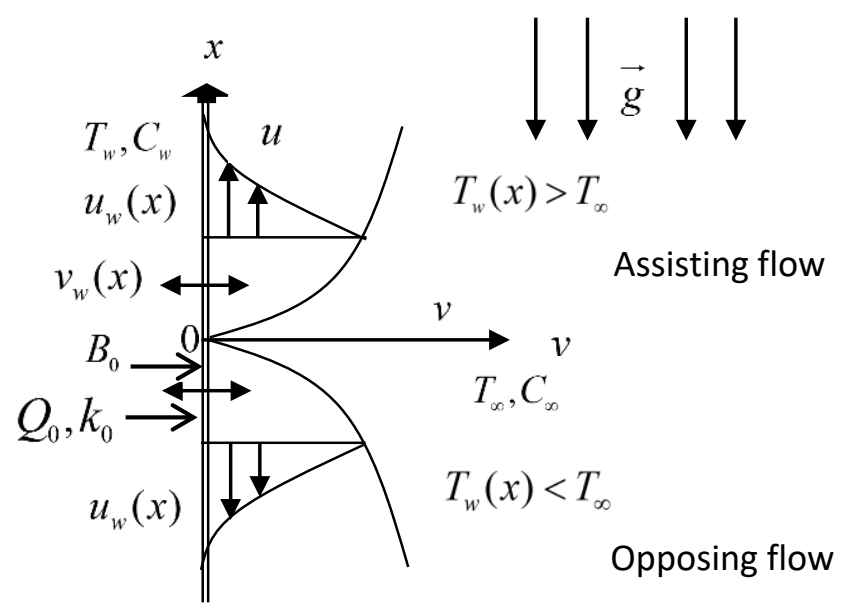

(a) Stretching sheet

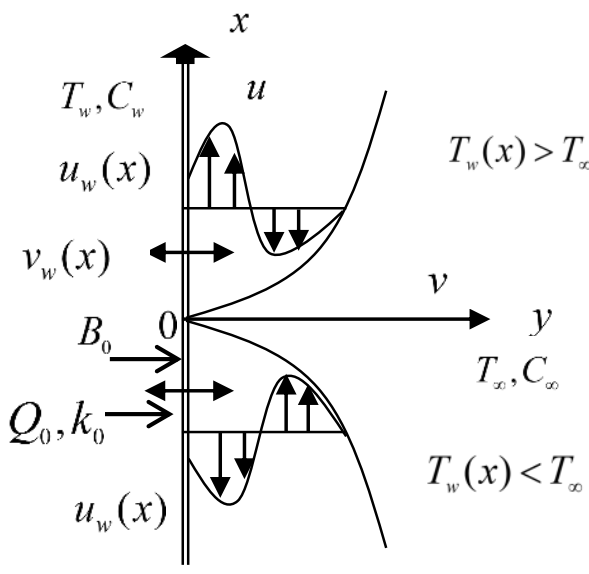

(b) Shrinking sheet

Fig. 1. Physical model and coordinate system: (a) Stretching sheet; (b) Shrinking sheet

The corresponding boundary conditions are:

$u=\varepsilon u_{w}, v=v_{w}, \omega=-m \frac{\partial u}{\partial y}, T=T_{w}, C=C_{w}$ as $y=0$

$u \rightarrow 0, \omega \rightarrow 0, T \rightarrow T_{\infty}, C \rightarrow C_{\infty}$ as $y \rightarrow \infty$

where $m$ is a positive constant between 0 and 1. As reported by Guram and Smith [9], the case $m=0$ represent strong concentration. Furthermore, the case $m=1 / 2$ is referred as weak concentration (Ahmadi [10]) while case $m=1$ is used to formulate the turbulent boundary layer flows modeling (Peddieson [11]). In addition, $v_{w}$ is a constant mass flux velocity. The parameter $\varepsilon$ is the stretching/shrinking parameter where $\varepsilon>0$ corresponds to the stretching case and $\varepsilon<0$ corresponds to the shrinking case.

The set of governing boundary layers Eqs. (1)-(5) in partial differential equation (PDE) form is reduced to set the coupled ordinary differential equation (ODE) by using the following similarity transformation 


$$
\begin{aligned}
& \eta=(a / v)^{1 / 2} y, \psi=(a v)^{1 / 2} x f(\eta), \omega=a x(a / v)^{1 / 2} g(\eta), \\
& \theta=\left(T-T_{\infty}\right) /\left(T_{w}-T_{0}\right), \phi=\left(C-C_{\infty}\right) /\left(C_{w}-C_{0}\right),
\end{aligned}
$$

where $\eta$ is the similarity variable, $v$ is the kinematic viscosity and $\psi$ is the stream function defined as $u=\partial \psi / \partial y$ and $v=-\partial \psi / \partial x$, altogether satisfying Eq. (1). By substituting Eq. (8) in Eqs. (2)-(5), the following ODEs are obtained:

$$
\begin{aligned}
& (1+K) f^{\prime \prime \prime}-f^{\prime 2}+f f^{\prime \prime}+K g^{\prime}-M f^{\prime}+\lambda \theta+N \phi=0, \\
& \left(1+\frac{K}{2}\right) g^{\prime \prime}-f^{\prime} g+f g^{\prime}-K\left(2 g+f^{\prime \prime}\right)=0, \\
& \frac{1}{\operatorname{Pr}} \theta^{\prime \prime}+\left[f \theta^{\prime}-\left(\theta+\delta_{1}\right) f^{\prime}+Q \theta\right]=0, \\
& \frac{1}{S c} \phi^{\prime \prime}+\left[f \phi^{\prime}-\left(\phi+\delta_{2}\right) f^{\prime}-C r \phi\right]=0 .
\end{aligned}
$$

Here, prime denote differentiation with respect to the similarity variable $\eta, f(\eta)$ is the dimensionless stream function, $g(\eta), \theta(\eta)$ and $\phi(\eta)$ are the dimensionless angular velocity, the dimensionless temperature and the dimensionless concentration of the fluid in the boundary layer region, respectively. The parameter $K=\kappa / \mu$ is the material parameter where $K=0$ and $K>0$ represent Newtonian fluid and micropolar fluid. The parameter $M=\sigma_{M} B_{0}^{2} / \rho a$ is the magnetic field parameter, $Q=Q_{0} / \rho C_{p} a$ is the heat source parameter, $\lambda=G r / \operatorname{Re}_{x}^{2}$ is the thermal buoyancy parameter with $\lambda>0$ corresponding to the assisting flow and $\lambda<0$ refers to the opposing flow, $N=G r^{*} / \operatorname{Re}_{x}^{2} N$ is the solutal buoyancy parameter where $G r=g \beta_{T} \Delta T x^{3} / v^{2}$ is the local Grashof number due to temperature and $G r^{*}=g \beta_{C} \Delta C x^{3} / v^{2}$ is the local Grashof number due to concentration. The parameter $\operatorname{Pr}=v / \alpha$ is the Prandtl number, $\delta_{1}=H / A$ is the thermal stratification parameter, $\delta_{2}=P / E$ refers to the solutal stratification parameter, $S c=v / D$ is the Schmidt number and $\mathrm{Cr}=k_{0} / a$ is the chemical reaction parameter.

Following the similarity transformation from Eq. (8), the associated boundary condition in Eqs. (6) and (7) turn into:

$$
\begin{aligned}
& f(0)=S, f^{\prime}(0)=\varepsilon, g(0)=-m f^{\prime \prime}(0), \theta(0)=1-\delta_{1}, \phi(0)=1-\delta_{2}, \\
& f^{\prime}(\eta) \rightarrow 0, g(\eta) \rightarrow 0, \theta(\eta) \rightarrow 0, \phi(\eta) \rightarrow 0
\end{aligned}
$$

where $S=v_{w} /-(a v)^{1 / 2}$ is the suction parameter with $v_{w}<0(S>0)$ for suction and $v_{w}>0(S<0)$ for injection.

The quantities of interest in this study are 
$C_{f}=\frac{\tau_{w}}{\rho u_{w}^{2}}, M_{w}=\frac{m_{w}}{\rho x u_{w}^{2}}, N u_{x}=\frac{x q_{w}}{k\left(T_{w}-T_{0}\right)}, S h_{x}=\frac{x q_{m}}{D_{B}\left(C_{w}-C_{0}\right)}$

where $k, \tau_{w}, m_{w}, q_{w}$ and $q_{m}$ are fluid thermal conductivity, the wall shear stress, the wall couple stress, the surface heat flux and the surface mass flux, respectively as given

$$
\tau_{w}=\left[(\mu+\kappa)\left(\frac{\partial u}{\partial y}\right)+\kappa \omega\right]_{y=0}, m_{w}=-\gamma\left(\frac{\partial \omega}{\partial y}\right)_{y=0}, q_{w}=-k\left(\frac{\partial T}{\partial y}\right)_{y=0}, q_{m}=-D\left(\frac{\partial C}{\partial y}\right)_{y=0}
$$

By substituting Eq. (15) into Eq. (14), we obtain the reduced skin friction coefficient $\operatorname{Re}_{x}^{1 / 2} C_{f}$, the local Nusselt number $\operatorname{Re}_{x}^{-1 / 2} N u_{x}$ and the local Sherwood number $\operatorname{Re}_{x}^{-1 / 2} S h_{x}$ as follow:

$\operatorname{Re}_{x}^{1 / 2} C_{f}=(1+(1-m) K) f^{\prime \prime}(0), \operatorname{Re}_{x} M_{w}=\left(1+\frac{K}{2}\right) g^{\prime}(0)$,

$\operatorname{Re}_{x}^{-1 / 2} N u_{x}=-\theta^{\prime}(0), \operatorname{Re}_{x}^{-1 / 2} S h_{x}=-\phi^{\prime}(0)$.

\section{Results and Discussion}

The nonlinear ordinary differential equations in Eqs. (9)-(12) accompanying the boundary equations in Eq. (13) are solved numerically with assistance of bvp4c solver in MATLAB software. For the purpose of obtaining the mathematical results, we have fixed the values of several parameters $K=M=N=1, \operatorname{Pr}=7, \lambda=-1, S c=0.78, \delta_{1}=0.1, \delta_{2}=0.2, S=2$ and $m=0$. Numerical results were obtained for different values $\varepsilon, C r$ and $Q$ in order to see their influence on the flow field and the quantities of physical interest in the presents study. Table 1 elucidates the values of $-\theta^{\prime}(0)$ and $-\theta^{\prime}(0)$ highlighted in the paper by Srinivasacharya and Upendar [5], and Khashi'ie et al., [7] for different values of $M$ for $K=N=\lambda=\operatorname{Pr}=1, \delta_{1}=0.1, S c=\delta_{2}=0.2, S=\varepsilon=m=0$ and $\eta_{\infty}=25$ in Eqs. (9)-(13). The comparison is drawn by neglecting the heat source and chemical reaction effects (by setting $Q=0$ and $C r=0$ in Eqs. (11)-(12)). The comparison is in excellent agreement and leads to the confidence of the present numerical results to be reported further. Further, Tables 2 and 3 tabulated the values of $f^{\prime \prime}(0), g^{\prime}(0),-\theta^{\prime}(0)$ and $-\phi^{\prime}(0)$ for different values of $C r$ and $Q$ when $K=M=N=1, \operatorname{Pr}=7, \lambda=-1, S c=0.78, \delta_{1}=0.1, \delta_{2}=0.2, S=2, m=0$ and $\varepsilon=-2$. 


\section{Table 1}

Comparison values of $-\theta^{\prime}(0)$ and $-\phi^{\prime}(0)$ for different values of $M$ for $K=N=\lambda=\operatorname{Pr}=1, \delta_{1}=0.1, S c=\delta_{2}=0.2, S=\varepsilon=m=0$ and $\eta_{\infty}=25$ in the absent of heat source and chemical reaction effects $(Q=0, C r=0)$

\begin{tabular}{|c|c|c|c|c|c|c|}
\hline & $\begin{array}{l}\text { Khashi'ie } \\
\text { et al., [7] }\end{array}$ & $\begin{array}{l}\text { Srinivasacharya } \\
\text { \& Upendar [5] }\end{array}$ & $\begin{array}{l}\text { Present } \\
\text { result }\end{array}$ & $\begin{array}{l}\text { Khashi'ie et } \\
\text { al., [7] }\end{array}$ & $\begin{array}{l}\text { Srinivasacharya } \\
\text { \& Upendar [5] }\end{array}$ & $\begin{array}{l}\text { Present } \\
\text { result }\end{array}$ \\
\hline$M$ & & $-\theta^{\prime}(0)$ & & & $-\phi^{\prime}(0)$ & \\
\hline 0 & 0.62576 & 0.62289 & 0.625761 & 0.28162 & 0.28042 & 0.281625 \\
\hline 0.5 & & & 0.589294 & & & 0.258241 \\
\hline 1 & 0.55941 & 0.55703 & 0.559410 & 0.23958 & 0.23895 & 0.239576 \\
\hline 1.5 & & & 0.534243 & & & 0.224371 \\
\hline 2 & 0.51257 & 0.51043 & 0.512573 & 0.21172 & 0.21176 & 0.211721 \\
\hline 2.5 & & & 0.493592 & & & 0.201000 \\
\hline 3 & & & 0.476742 & & & 0.191774 \\
\hline 3.5 & & & 0.461623 & & & 0.183728 \\
\hline 4 & & & 0.447938 & & & 0.176634 \\
\hline 4.5 & & & 0.435460 & & & 0.170320 \\
\hline 5 & & & 0.424013 & & & 0.164654 \\
\hline 10 & & & 0.344640 & & & 0.128504 \\
\hline
\end{tabular}

\section{Table 2}

The values of $f^{\prime \prime}(0), g^{\prime}(0),-\theta^{\prime}(0)$ and $-\phi^{\prime}(0)$ for different values of $Q$ when $K=M=N=1, \operatorname{Pr}=7, \lambda=-1, S c=0.78, \delta_{1}=C r=0.1, \delta_{2}=0.2, S=2, m=0$ and $\varepsilon=-2$

\begin{tabular}{lllll}
\hline$Q$ & $f^{\prime \prime}(0)$ & $g^{\prime}(0)$ & $-\theta^{\prime}(0)$ & $-\phi^{\prime}(0)$ \\
\hline 0 & 2.003328 & -1.051363 & 8.319752 & -0.936619 \\
0.1 & 2.020590 & -1.052411 & 8.871766 & -0.906988 \\
0.2 & 2.056005 & -1.054243 & 10.171305 & -0.855729 \\
0.3 & 2.117810 & -1.057211 & 12.587756 & -0.776629 \\
0.4 & 2.208256 & -1.061485 & 16.251232 & -0.672434 \\
\hline
\end{tabular}

\section{Table 3}

The values of $f^{\prime \prime}(0), g^{\prime}(0),-\theta^{\prime}(0)$ and $-\phi^{\prime}(0)$ for different values of $C r$ when $K=M=N=1, \operatorname{Pr}=7, \lambda=-1, S c=0.78, \delta_{1}=Q=0.1, \delta_{2}=0.2, S=2, m=0$ and $\varepsilon=-2$

\begin{tabular}{lllll}
\hline$C r$ & $f^{\prime \prime}(0)$ & $g^{\prime}(0)$ & $-\theta^{\prime}(0)$ & $-\phi^{\prime}(0)$ \\
\hline 0 & 1.937599 & -1.040979 & 8.424528 & -0.779911 \\
0.1 & 2.020590 & -1.052411 & 8.871766 & -0.906988 \\
0.2 & 2.104432 & -1.064297 & 9.224810 & -1.049855 \\
0.3 & 2.190522 & -1.076744 & 9.512989 & -1.209996 \\
0.4 & 2.279882 & -1.089842 & 9.754033 & -1.389385 \\
\hline
\end{tabular}

Influences of heat source $Q$ on fluid velocity $f^{\prime}(\eta)$, temperature $\theta(\eta)$ and concentration $\phi(\eta)$ are demonstrated in Figures 2 - 4. From Figure 2, it is seems that the fluid velocity is increased as the values of the heat source parameter $Q$ arises. This trend is obtained because heat source increases the momentum boundary layer thickness as portrayed in Figure 2. 


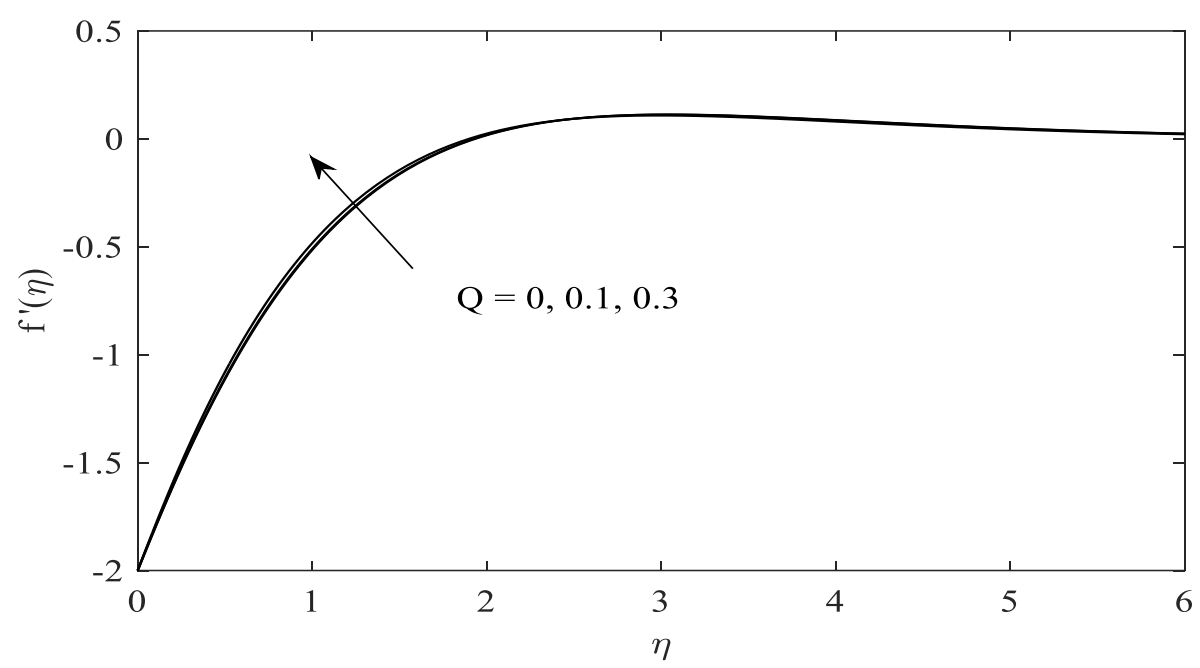

Fig. 2. The velocity profile $f^{\prime}(\eta)$ for different values of $Q$ with $\lambda=-1$,

$K=M=N=1, \operatorname{Pr}=7, S c=0.78, \delta_{1}=C r=0.1, \delta_{2}=0.2, S=2, m=0$ and $\varepsilon=-2$

The impact of the heat source parameter on the fluid temperature is elucidated in Figure 3 . It is concluded that the temperature and the thermal boundary layer thickness decrease when the heat source arises.

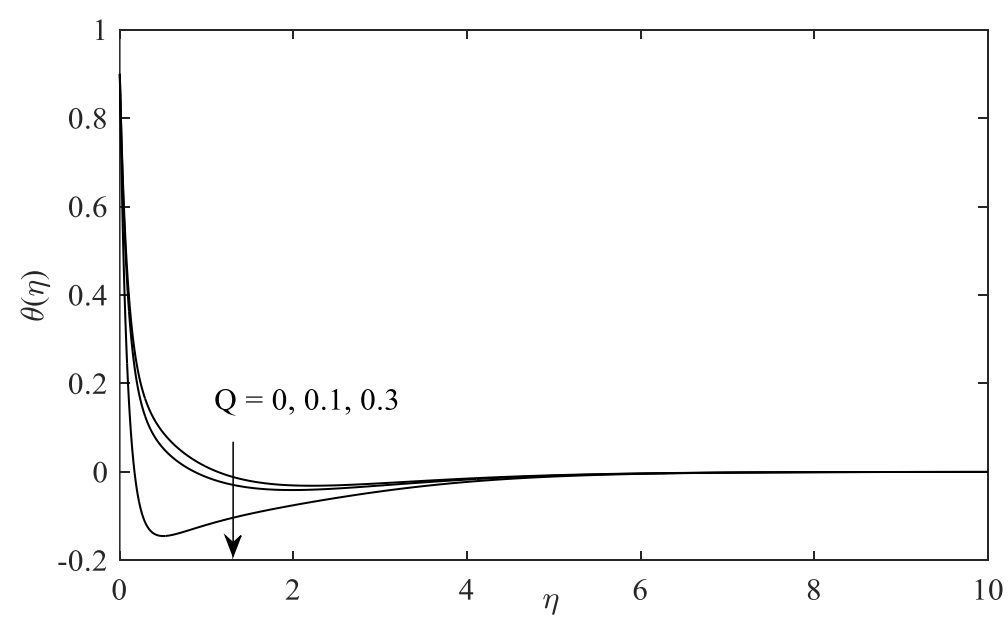

Fig. 3. The temperature profile $\theta(\eta)$ for different values of $Q$ with $\lambda=-1, K=M=N=1, \operatorname{Pr}=7, S c=0.78, \delta_{1}=$ $\mathrm{Cr}=0.1, \delta_{2}=0.2, S=2, m=0$ and $\varepsilon=-2$

From Figure 4, it is observed that an increase in the heat source parameter creates a reduction in the fluid concentration. This situation happens due to the heat source effect which leads to the reduction in the concentration boundary layer thickness by increasing the values of $Q$. 


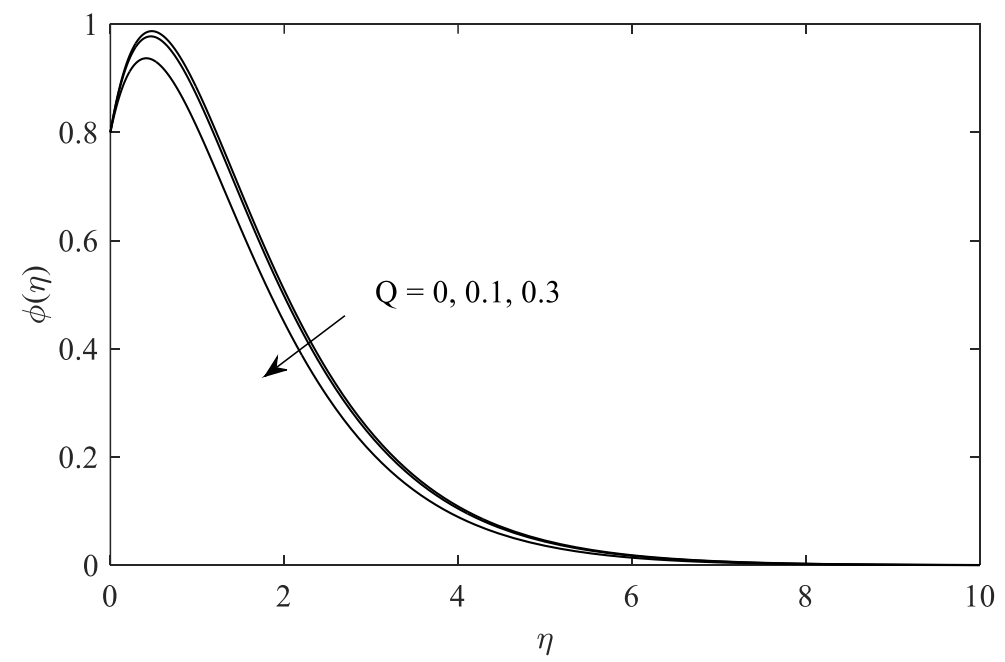

Fig. 4. The concentration profile $\phi(\eta)$ for different values of $Q$ with $\lambda=-1, K=M=N=1, \operatorname{Pr}=7, S c=0.78, \delta_{1}=$ $\mathrm{Cr}=0.1, \delta_{2}=0.2, S=2, m=0$ and $\varepsilon=-2$

Figures 5-7 display the variation of $f^{\prime \prime}(0),-\theta^{\prime}(0)$ and $-\phi^{\prime}(0)$ with stretching/shrinking parameter $\varepsilon$ for different values of $C r$ for the opposing flow $(\lambda=-1)$, respectively. The critical values of $\varepsilon$ denoted by $\varepsilon_{c}$ for $Q=0,0.1$ and 0.3 are $\varepsilon_{c}=-2.3305,-2.2617$ and -2.1643 in a way that the similarity solution of ODEs in Eqs. (9)-(13) exists.

It is clear in Figure 5 that the heat source effect increases the velocity gradient $f^{\prime \prime}(0)$ and as a result, the skin friction coefficient along the surface of the vertical sheet increases. This phenomenon occurs because of the increased rate of velocity with the heat source parameter. This finding is consistent with the graph presented in Figure 2.

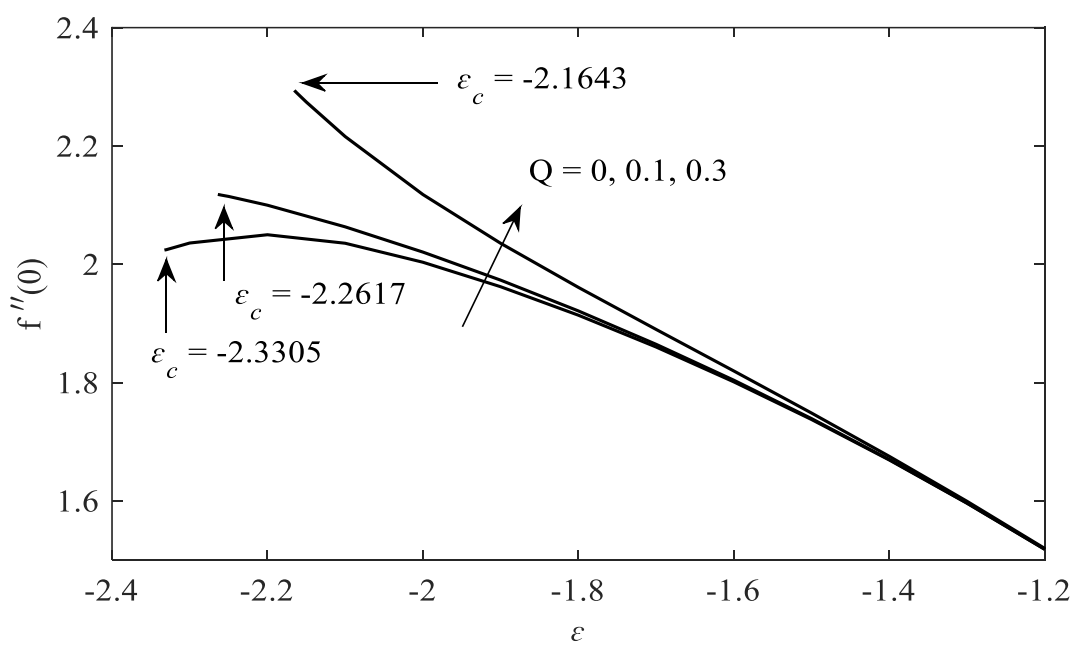

Fig. 5. Variation of $f^{\prime \prime}(0)$ with $\varepsilon$ for different values of $Q$ with $\lambda=-1, K=M=N=1, \operatorname{Pr}=7, S c=0.78, \delta_{1}=C r=$ $0.1, \delta_{2}=0.2, S=2, m=0$ 
Figure 6 is sketched to see the effect of the heat source on the temperature gradient $-\theta^{\prime}(0)$. Imposing the heat source effect leads to an increase in the temperature gradient $-\theta^{\prime}(0)$ and in turn increasing the local Nusselt number which represents the heat transfer rate at the surface.

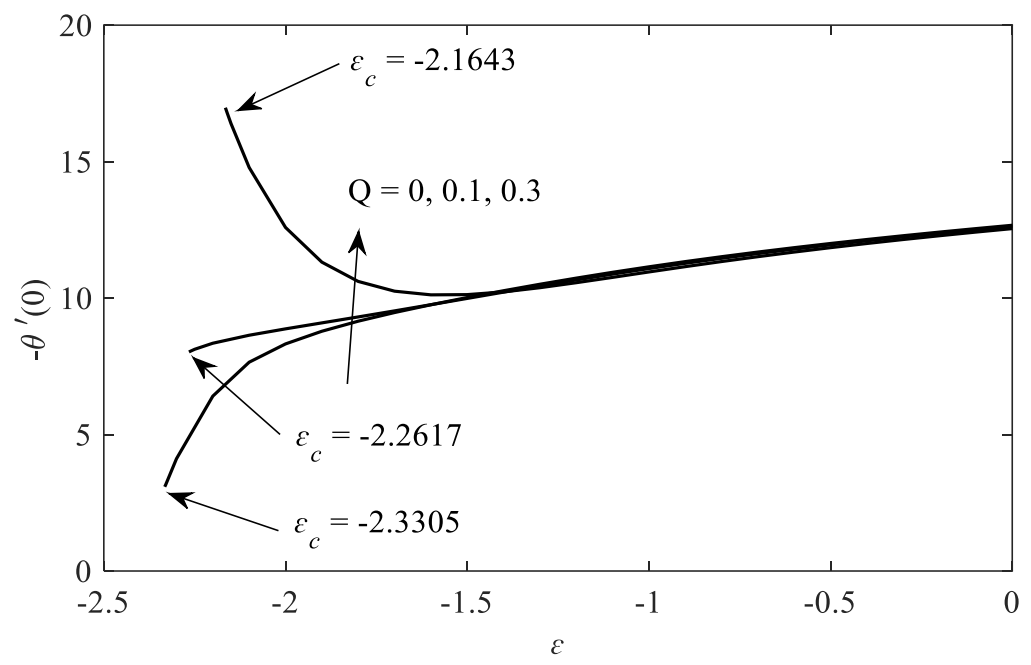

Fig. 6. Variation of $-\theta^{\prime}(0)$ with $\varepsilon$ for different values of $Q$ with $\lambda=-1, K=M=N=1, \operatorname{Pr}=7, S c=0.78, \delta_{1}=C r=$ $0.1, \delta_{2}=0.2, S=2, m=0$

On the other hand, the magnitude of the concentration gradient $-\phi^{\prime}(0)$ is reduced by imposing the heat source effect as illustrated in Figure 7. These findings Figures 5-7 are supported by the values of $f^{\prime \prime}(0),-\theta^{\prime}(0)$ and $-\phi^{\prime}(0)$ presented in Table 2.

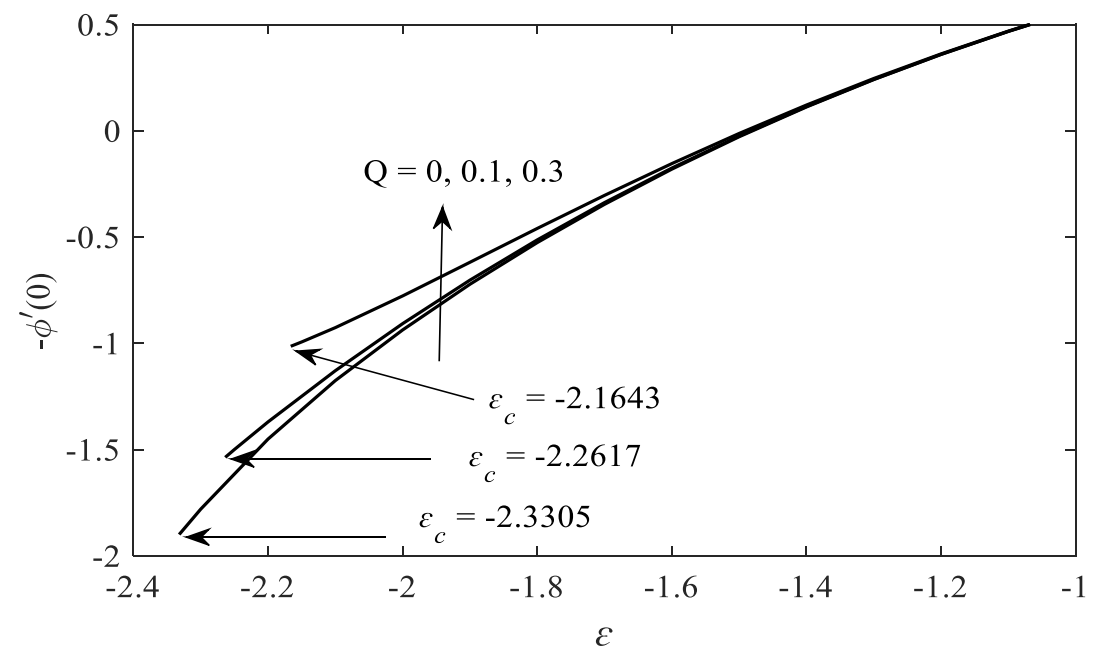

Fig. 7. Variation of $-\phi^{\prime}(0)$ with $\varepsilon$ for different values of $Q$ with $\lambda=-1, K=M=N=1, \operatorname{Pr}=7, S c=0.78, \delta_{1}=C r=0.1, \delta_{2}=$ $0.2, S=2, m=0$

Figures 8-10 are drawn to investigate the influence of the chemical reaction parameter $\mathrm{Cr}$ on the profiles of velocity $f^{\prime}(\eta)$, temperature $\theta(\eta)$ and concentration $\phi(\eta)$ of the fluid, respectively. Similar to heat source effect on velocity, it is found that the rise in the value of the chemical reaction has increased the velocity of the fluid as shown in Figure 8. This phenomenon occurs as the 
momentum boundary layer thickness becomes thinner as the chemical reaction effect is imposed in the flow and in turn increases the value of the velocity gradient at the surface. As a result, the skin friction coefficient at the surface increases as can be seen in Figure 11.

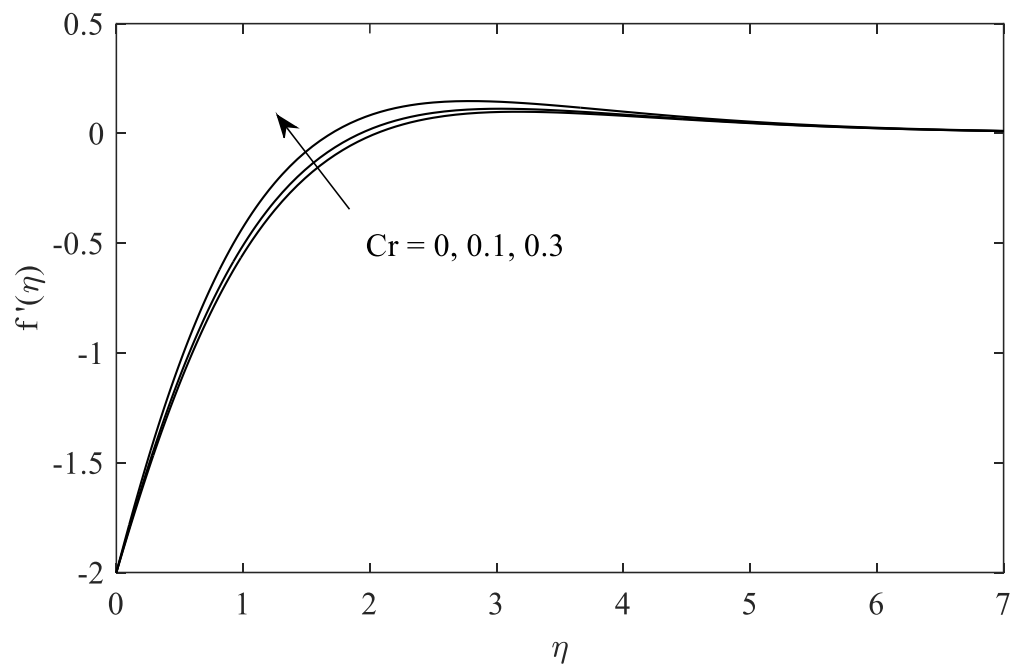

Fig. 8. The velocity profile $f^{\prime}(\eta)$ for different values $\mathrm{Cr}$ of with $\lambda=-1, K=M=N=1, \operatorname{Pr}=7, S c=0.78, \delta_{1}=Q=$ $0.1, \delta_{2}=0.2, S=2, m=0$ and $\varepsilon=-2$

The curves of the temperature profile for some values of $\mathrm{Cr}$ are shown in Figure 9. It can be assured that the increasing chemical reaction strength has decreased the dimensionless temperature. As the consequence, the thermal boundary layer thickness decreases and hence enhances the heat transfer rate at the surface as depicted in Figure 12.

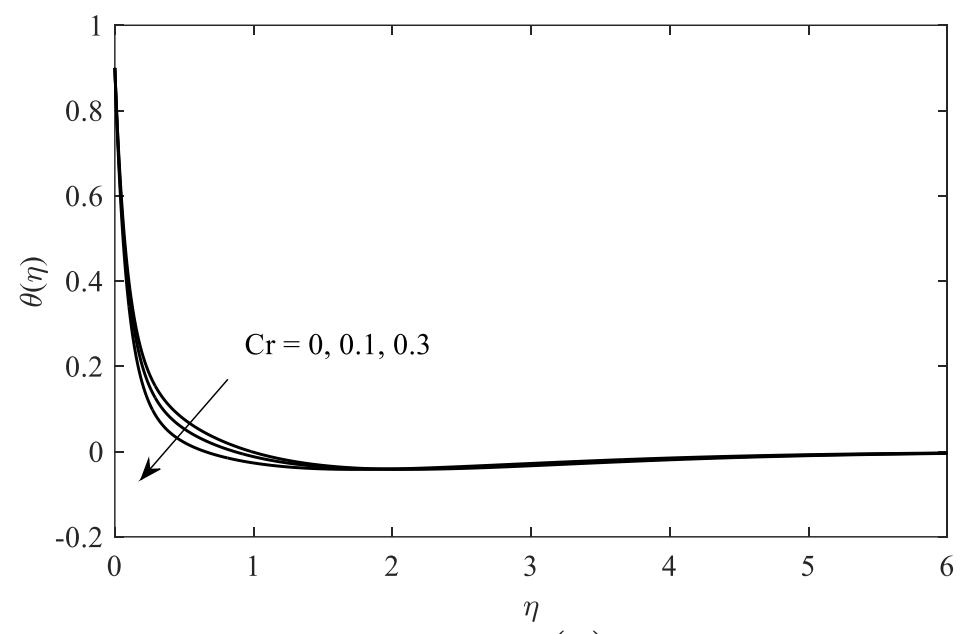

Fig. 9. The temperature profile $\theta(\eta)$ for different values

$C r$ of with $\lambda=-1, K=M=N=1, P r=7, S c=$

$0.78, \delta_{1}=Q=0.1, \delta_{2}=0.2, S=2, m=0$ and $\varepsilon=-2$

The influence of the chemical reaction on the concentration profile is delineated in Figure 10. It can be noted that imposing chemical reaction leads to an additional heat to the fluid flow and hence enhances the concentration as portrayed in Figure 10. 


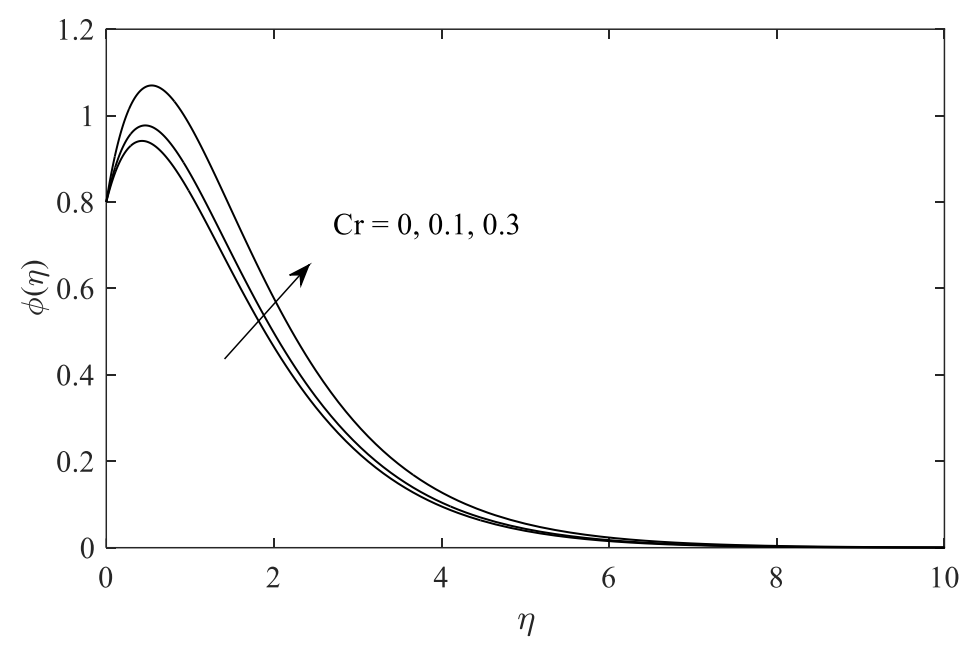

Fig. 10. The concentration profile $\phi(\eta)$ for different values

$C r$ of with $\lambda=-1, K=M=N=1, \operatorname{Pr}=7, S c=$

$0.78, \delta_{1}=Q=0.1, \delta_{2}=0.2, S=2, m=0$ and $\varepsilon=-2$

Figures 11-13 are plotted in order to see the impacts of the chemical reaction parameter on the velocity gradient at the surface, $f^{\prime \prime}(0)$, the temperature gradient at the surface, $-\theta^{\prime}(0)$ and the concentration gradient at the surface, $-\phi^{\prime}(0)$. These Figures 11-13 are directly representing the skin friction coefficient, the local Nusselt number and the local Sherwood number, respectively.

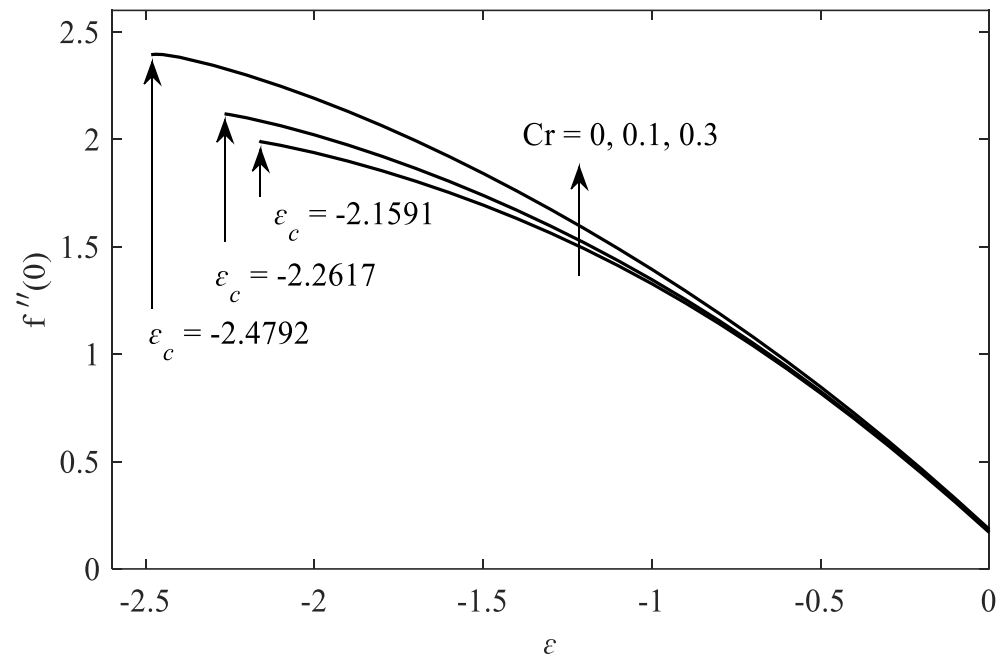

Fig. 11. Variation of $f^{\prime \prime}(0)$ with $\varepsilon$ for different values of

$C r$ with $\lambda=-1, K=M=N=1, \operatorname{Pr}=7, S c=0.78, \delta_{1}=$ $Q=0.1, \delta_{2}=0.2, S=2, m=0$

It is observed that as the chemical reaction parameter increases, the skin friction coefficient, the local Nusselt number and the magnitude of the local Sherwood number increase. This is because as the chemical reaction parameter increases, the velocity and concentration increase (see Figures 8 \& 10) while the temperature act in the opposite manner (see Figure 9). As a result, the skin friction, heat, and mass transfer at the surface are enhanced by way of imposing the chemical reaction parameter as illustrated in Figures 11-13. Finally, it is important to highlight that all the velocity, temperature and concentration distributions are presented in Figures 2-4 and Figures 8-10 satisfy the 
far field boundary conditions in Eq. (13) asymptotically and indirectly leading to the confidence to the present numerical results obtained.

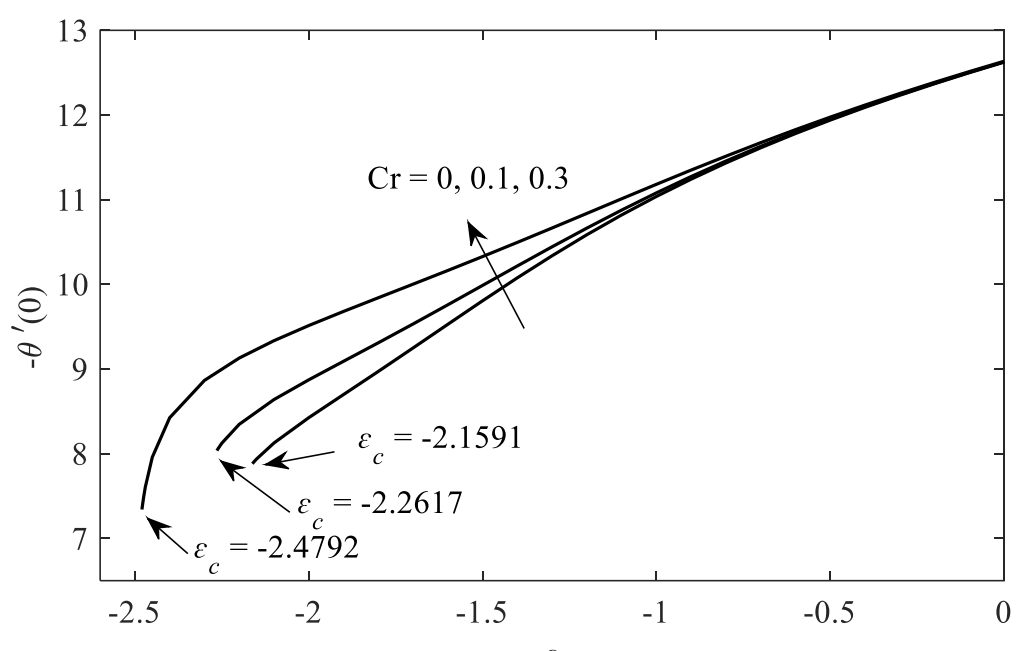

Fig. 12. Variation of $-\theta^{\prime}(0)$ with $\varepsilon$ for different values of $C r$ with $\lambda=-1, K=M=N=1, \operatorname{Pr}=7, S c=0.78, \delta_{1}=$ $Q=0.1, \delta_{2}=0.2, S=2, m=0$

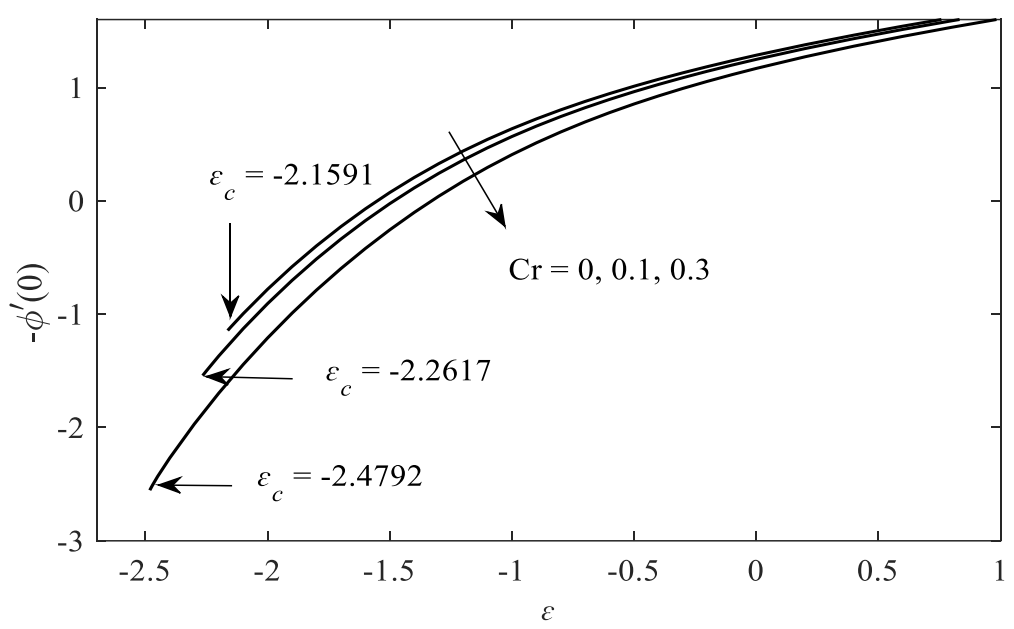

Fig. 13. Variation of $-\phi^{\prime}(0)$ with $\varepsilon$ for different values of $\mathrm{Cr}$ with $\lambda=-1, K=M=N=1, \operatorname{Pr}=7, S c=0.78, \delta_{1}=Q=$ $0.1, \delta_{2}=0.2, S=2, m=0$

\section{Conclusions}

A steady MHD micropolar fluid flow on double stratification over a permeable a stretching/shrinking vertical sheet with the existence of chemical reaction and heat source effects are studied. The investigations have been conducted numerically by using bvp4c solver in MATLAB software. The present numerical results have also been compared with the published work for limited case and the comparison are in good agreement. The major outcomes of the present study are as follows:

I. Fluid velocity increases while both the temperature and concentration decrease due to the heat source effect. 
II. Both velocity and concentration of the fluids increase with the increasing of chemical reaction parameter while the fluid temperature acts in the opposite behavior.

III. The skin friction coefficient and local Nusselt number which represent the heat transfer rate at the surface are enhanced in the presence of the chemical reaction and heat source effect.

IV. In contrast, the magnitude of the Sherwood number which represents the mass transfer rate at the surface decreases as the heat source parameter arises while it increases with the chemical reaction effect.

\section{Acknowledgement}

The author would like to acknowledge the support from the Fundamental Research Grant Scheme (FRGS) under a grant number of FRGS/1/2018/STG06/UNIMAP/02/3 from the Ministry of Education Malaysia.

\section{References}

[1] Eringen, A.C. "Theory of micropolar fluids." J. Math. Mech. 16(1966): 1-18. https://www.jstor.org/stable/24901466

[2] Eringen, A.C. "Theory of thermomicropolar fluids." J. Math. Anal. Appl. 38(1972): 480-496. https://doi.org/10.1016/0022-247X(72)90106-0

[3] Ahmad, Kartini, Anuar Ishak, and Roslinda Nazar. "Micropolar fluid flow and heat transfer over a nonlinearly stretching plate with viscous dissipation." Mathematical Problems in Engineering, 2013 (2013): Article no. 257161. https://doi.org/10.1155/2013/257161

[4] Lukaszewicz, G. Micropolar Fluids Theory and Applications, Birkhauser, Basel, 1999.

[5] Srinivasacharya, D. and Mendu Upendar. "Effect of double stratification on MHD free convection in a micropolar fluid." J. Egypt. Math. Soc. 21, no. 3 (2013): 370-378.

https://doi.org/10.1016/i.joems.2013.02.006

[6] Rashad, A.M., S. Abbasbandy, and Ali, J. Chamkha, "Mixed convection flow of a micropolar fluid over a continuously moving vertical surface immersed in a thermally and solutally stratified medium with chemical reaction." Journal of the Taiwan Institute of Chemical Engineers 45, no. 5 (2014): 2163-2169.

https://doi.org/10.1016/j.jtice.2014.07.002

[7] Khashi'ie, Najiyah Safwa, Norihan Md Arifin, Roslinda Nazar, Ezad Hafidz Hafidzuddin, Nadihah Wahi, and Ioan Pop. "Mixed convective flow and heat transfer of a dual stratified micropolar fluid induced by a permeable stretching/shrinking sheet." Entropy 21, no. 12 (2019): 1162.

https://doi.org/10.3390/e21121162

[8] Ibrahim, S. Mohammed and K. Suneetha. "Heat source and chemical effects on MHD convection flow embedded in a porous medium with Soret, viscous and Joules dissipation." Ain Shams Engineering Journal 7, no. 2 (2016): 811818. https://doi.org/10.1016/i.asej.2015.12.008

[9] Guram, G.S. and A.C. Smith. "Stagnation flows of micropolar fluids with strong and weak interactions." Comp. Math. Appl. 6, no. 2 (1980): 213-233.

https://doi.org/10.1016/0898-1221(80)90030-9

[10] Ahmadi, Goodarz. "Self-similar solution of imcompressible micropolar boundary layer flow over a semi-infinite plate." Int. J. Eng. Sci. 14, no. 7 (1976): 639-646. https://doi.org/10.1016/0020-7225(76)90006-9

[11] Peddieson, J. Jr. "An application of the micropolar fluid model to the calculation of a turbulent shear flow." Int. J. Eng. Sci. 10, no. 1 (1972): 23-32.

https://doi.org/10.1016/0020-7225(72)90072-9 